\title{
Análisis temático de los proyectos de fin de carrera del título de Licenciado en Documentación de la Universidad de Murcia
}

\author{
Subject analysis of the final projects of the Documentation programme in the University of Murcia
}

\author{
Francisco Javier MARTínez MÉnDeZ (1) y Rosana LóPEZ CARREÑo (2) \\ Facultad de Comunicación y Documentación de la Universidad de Murcia, \\ Campus de Espinardo s/n 30100 Murcia, (1) javima@um.es (2) rosanalc@um.es
}

\begin{abstract}
Resumen
La adaptación al EEES de la Universidad Española implica una amplia serie de cambios en los actuales planes de estudio de Documentación. La puesta en marcha del nuevo título de Grado de Información y Documentación implica la puesta en marcha de una asignatura obligatoria denominada "Proyecto Fin de Grado" con la idea de introducir al estudiante a la realización de unos proyectos de investigación básica o aplicada sobre alguna de las materias estudiadas a lo largo de la carrera. Esta asignatura no es nueva en la Universidad de Murcia donde se imparte desde el curso 1998/1999, habiéndose desarrollado una amplia serie de proyectos desde entonces. El objetivo de este trabajo es analizar los temas principales de cada uno de ellos y verificar su grado de concordancia con las materias del plan de estudios vigente y con la propuesta de materias obligatorias del Libro Blanco del nuevo título, verificando si, al final de los estudios, los autores de estos proyectos encauzan su actividad dentro de las materias principales del plan de estudios o si, por el contrario, orientan su investigación hacia materias optativas e, incluso, materias que aparecen de forma colateral en sus curricula. Esta revisión proporciona a los responsables de la nueva asignatura una información muy valiosa con vistas a su puesta en marcha.
\end{abstract}

Palabras clave: Planes de estudio. Espacio Europeo de Educación Superior. Graduado en información y documentación. Proyecto fin de grado.

\section{Introducción}

Una de las novedades que introduce el Real Decreto $1393 / 2007$, de 30 de octubre que regula la ordenación de las enseñanzas universitarias con vistas a la entrada en vigor del Espacio Europeo de Educación Superior a partir del curso 2010/2011 (MEC, 2007) es la realización "con carácter obligatorio" de un Trabajo Fin de Grado que tendrá una "tendrá una extensión mínima de 6 y máxima de 30 créditos ECTS" y "deberá realizarse en la fase final del plan de

\begin{abstract}
The adaptation to EHEA of the Spanish University involves a wide range of changes in the existing curricula of Documentation. The launch of the new title 'Bachelor in Information and Documentation' implies the implementation of a compulsory subject -the Grade Thesisoriented to the realization of a research project at the end of the studies, with the objective of introducing the student in basic or applied research about any of the subjects studied along the grade. The object of this paper is to analyze the main themes of the projects developed in the LIS studies of the University of Murcia; and verify their degree of consistency with the subjects of the present curriculum and with the proposed compulsory subjects for the new title; checking whether if, at the end of their studies, the students channelled their activity within the main subjects of the curriculum or whether they directed their research into elective subjects and even into collateral subjects of their curricula. This review provides very valuable information with a view to this launching.
\end{abstract}

Keywords: Course programme. European Higher Education Area. Information Science. Documentation. Grade thesis.

estudios y deberá estar orientado a la evaluación de competencias asociadas a la titulación". Esta asignatura se presenta como una novedad en los planes de estudios que ya se ponen en marcha en el curso 2008/2009 (Carlos III de Madrid, Salamanca y Zaragoza ) y en los que les seguirán. Si bien este no es el caso de la Universidad de Murcia, donde existe esta asignatura desde que se imparte el título (a extinguir) de Licenciado en Documentación y donde se han desarrollado ya más de 250 trabajos de 
esta índole a lo largo de 9 cursos académicos. Tal como indica el reglamento de desarrollo de este Trabajo Fin de Carrera, el mismo "se entiende como el resultado final de un proyecto o trabajo personal del alumno, donde manifieste los conocimientos y aptitudes adquiridos a los largo de la titulación de Licenciado en Documentación. El tema de realización del PFC ha debido ser propuesto y aceptado de antemano, debiendo estar relacionado con los contenidos del Plan de Estudios de la Licenciatura en Documentación" (UMU, 2001, p. 1).

Tanto el volumen de trabajo desarrollado como el tiempo que esta asignatura lleva vigente parecen suficientes para estudiar cuáles han sido los temas de interés más frecuentemente abordados por los estudiantes y de paso contrastar si estos temas se encuentran directamente vinculados a la distribución de materias en el plan de estudios, intentando verificar si todas las materias resultan de igual interés para los estudiantes, o si, por el contrario, se decantan más por unas que por otro, o, incluso, si la temática de sus trabajos se centra más en asignaturas optativas. Este recuento nos permitirá determinar en qué medida han influido las directrices generales del título en la elección de los estudiantes.

Pero el experimento no acaba en este análisis, porque de forma paralela se puede intentar extrapolar el experimento al nuevo espacio determinado por los planes de estudio adaptados al EEES. Así, se puede repetir la comparación tomando ahora como muestra la propuesta del Libro Blanco de ANECA de Título de Grado en Información y Documentación (ANECA, 2005). De esta forma se podrá percibir si la distribución de los trabajos realizados se adapta más o menos a las materias de interés en el plan de estudios en relación con el actual título de Licenciado en Documentación.

Esto puede resultar de interés para todos aquellos centros que van a poner en marcha esta asignatura con el fin de introducir las medidas correctoras que consideren (si fuera el caso) para el desarrollo de esta nueva asignatura, que además de obligatoria realización representará el culmen del proceso de enseñanza/aprendizaje de los estudios dentro de este nuevo contexto, ya que para su realización se precisará haber superado el resto de los créditos del plan de estudios.

\section{La troncalidad en el Plan de Estudios de Licenciado en Documentación}

A la hora de confeccionar el Plan de Estudios de Licenciado en Documentación (UMU, 2000), la
Facultad de Comunicación y Documentación (entonces de Ciencias de la Documentación) apostó por un bajo porcentaje de troncalidad en el plan de estudios. Las razones eran variadas, siendo la principal querer dejar plena elección al estudiante a la hora de configurar su curricula por medio de las cinco intensificaciones que se introdujeron en este título (Archivística, Documentación Científica y Técnica, Gestión de Instituciones Documentales, Gestión de la Información y Documentacion en la Empresa e Ingeniería de la Información). La plasmación de esta idea no hubiera sido posible de no ser por la colaboración de muchos departamentos universitarios que pusieron en marcha un importante conjunto de asignaturas optativas a tal fin. Transcurrida casi una década, se puede afirmar que el resultado ha sido positivo en cuanto a la libertad de configuración del currículo de los estudiantes, pero no tanto en lo que se refiere al aprovechamiento de las intensificaciones, aspecto escasamente aprovechado por los alumnos. ¿Cuál ha sido el motivo de no hacer uso de esta opción? La respuesta no es fácil aunque quizá el alto número de optativas exigidos para obtener una intensificación -ocho- lo ha dificultado, tanto por la temática de las mismas, que no siempre han resultado ser del gusto de los estudiantes, como por la oferta de las mismas, ya que desgraciadamente, en la práctica cotidiana de la docencia universitaria, no siempre los departamentos han podido ofrecer todas las asignaturas a lo largo de todos los cursos, habiendo desaparecido algunas y habiéndose ofertado otras de forma alterna.

Así, en nuestra Facultad, la troncalidad presente en el Plan de Estudios de Licenciado en Documentación es más o menos similar a la reflejada en las Directrices Generales de este título (Real Decreto 91/1992 de 17 de julio, BOE 206 de 27 de agosto de 1992, p. 29800-29802), quedando de la siguiente manera:

1. Administración de Recursos en Unidades Informativas (8 créditos).

2. Estadística (7 créditos).

3. Planificación y Evaluación de Sistemas de Información y Documentación (7 créditos).

4. Sistemas de Representación y Procesamiento Automático del Conocimiento (8 créditos).

5. Sistemas Informáticos (6 créditos).

6. Técnicas de Indización y Resumen en Documentación Científica (10 créditos).

7. Técnicas Documentales Aplicadas a la Investigación (6 créditos). 
Como asignatura obligatoria se incluyó la realización de un Proyecto Fin de Carrera (valorado en 6 créditos). Esta idea ha resultado a la larga pionera en los estudios de Información y Documentación a nivel nacional y constituye la base de este estudio.

\section{Las materias obligatorias en el Plan de Estudios de Graduado en Información y Documentación}

El Libro Blanco del Título de Grado en Información y Documentación (ANECA, 2005) ha representado una muy positiva experiencia de colaboración entre los centros universitarios españoles que imparten estas enseñanzas. El documento formó parte de la primera serie de Libros Blancos patrocinados por ANECA y aunque han sido muchos los cambios legislativos introducidos desde su aprobación (hacia el primer trimestre de 2004), sigue siendo un documento de base para la elaboración de los nuevos planes de estudio en el marco del EEES. En este documento se han considerado como materias obligatorias a estudiar para un Graduado en Información y Documentación las siguientes:

1. Documentos, Unidades y Sistemas de Información (12 créditos).

2. Planificación, Organización y Evaluación de Unidades de Información (24 créditos).

3. Fuentes de Información (12 créditos).

4. Representación y Recuperación de la información (36 créditos).

5. Gestión Técnica de Documentos de Archivo (18 créditos).

6. Tecnologías de la Información y Edición Digital (24 créditos).

7. Fundamentos y Metodologías de Investigación. Estudios Métricos de Información (18 créditos).

8. Practicum (12 créditos).

En el caso particular de cada Universidad, esta propuesta debe adaptarse a la nueva estructura de las enseñanzas universitarias que viene fijada por el Real Decreto 1393/2007, de 30 de octubre, por el que se establece la ordenación de las enseñanzas universitarias oficiales. Es en este decreto (MEC, 2007) donde se introduce la asignatura obligatoria de Trabajo Fin de Grado, objeto de este estudio. El resumen se debe redactar de forma clara y concisa, sin redundancias ni perífrasis ni información difícil de interpretar, como neologismos o abreviaturas que no se desarrollan.

\section{Metodología}

El primer consistía en recopilar información acerca del número de trabajos desarrollados y la temática de los mismos. El reglamento para el desarrollo de esta asignatura indica que debe depositarse una copia en la Biblioteca Universitaria (UMU, 2000, p. 4). Así, por medio de una búsqueda exhaustiva en la OPAC de la Biblioteca Universitaria de los PFC catalogados, se procedió a su listado para posteriormente clasificarlos de dos maneras: (1) por la materia del Plan de Estudios vigente para la obtención del título de Licenciado en Documentación más afín al tema del PFC y (2) por la materia obligatoria propuesta en el Libro Blanco del Título de Grado en Información y Documentación más afín al tema del PFC. En este proceso de clasificación se tomaron en cuenta únicamente los temas de cada trabajo desarrollado, independientemente de que el profesor director del proyecto imparta docencia en otra materia troncal (hecho que ocurre con cierta frecuencia). Asimismo, se procedió a la consignación del Área de Conocimiento a la que pertenece el director de cada PFC.

A continuación de describen las materias tratadas en los PFC:

\begin{tabular}{|c|c|}
\hline Sigla & Materia \\
\hline ESTA & Estadística \\
\hline OREMP & Organización de Empresas \\
\hline PESID & $\begin{array}{l}\text { Planificación de Servicios de } \\
\text { Información Documental }\end{array}$ \\
\hline SINFO & Sistemas informáticos \\
\hline SRPAC & $\begin{array}{l}\text { Representación y Organización del } \\
\text { Conocimiento }\end{array}$ \\
\hline TDAI & $\begin{array}{l}\text { Técnicas Documentales Aplicadas a } \\
\text { la Investigación }\end{array}$ \\
\hline TIN & Técnicas de Indización \\
\hline TMARI & $\begin{array}{l}\text { Técnicas y Métodos de } \\
\text { Recuperación de Información }\end{array}$ \\
\hline Tr.(Dipl.) & $\begin{array}{l}\text { Asignaturas Troncales del Título de } \\
\text { Diplomado en Biblioteconomía y } \\
\text { Documentación }\end{array}$ \\
\hline Op. (Dipl.) & $\begin{array}{l}\text { Asignaturas Optativas del Título de } \\
\text { Diplomado en Biblioteconomía y } \\
\text { Documentación }\end{array}$ \\
\hline Op. (Lic.) & $\begin{array}{l}\text { Asignaturas Optativas del Título de } \\
\text { Licenciado en Documentación }\end{array}$ \\
\hline
\end{tabular}

Tabla I. Materias del Plan de Estudios de Licenciado en Documentación consideradas en el estudio

Se puede observar en la tabla anterior que aparecen materias que no son troncales del Plan de Estudios de Licenciado en Documentación. Esto se debe a que tras analizar la temática de los 
proyectos realizados se observó que un número importante de ellos correspondían más a asignaturas troncales y optativas del título de Diplomado en Biblioteconomía y Documentación o a asignaturas optativas de la Licenciatura en Documentación (especialmente la asignatura Conservación y Restauración del Patrimonio Documental) que a las propias de este título. Por ello, debido a que este número de proyectos es relevante, se decidió incluir tres nuevas áreas temáticas de interés para el desarrollo del PFC, que se denominan (de forma genérica): (1) Troncales del Título de Diplomado en Biblioteconomía y Documentación, (2) Optativas del Título de Diplomado en Biblioteconomía y Documentación, y (3) Optativas del Título de Licenciado en Documentación.

También se intenta estudiar la relación entre las materias del plan actual de Licenciado y las materias a implantar con el nuevo plan de estudios de título de Grado, con el fin de verificar si la nueva distribución de materias obligatorias responde a las preferencias de los estudiantes de forma mayor o menor que la planteada hace unos años con el título de Licenciado, ya en camino de extinción. Por ello, se trabaja con las materias propuestas en el Libro Blanco del Título de Grado en Información y Documentación, donde aparecen siete materias obligatorias:

\begin{tabular}{|c|c|}
\hline Sigla & Materia \\
\hline $\mathrm{ARCHI}$ & $\begin{array}{l}\text { Gestión Técnica de Documentos de } \\
\text { Archivo }\end{array}$ \\
\hline DOCU & $\begin{array}{l}\text { Documentos, Unidades y Sistemas } \\
\text { de Información }\end{array}$ \\
\hline FUEN & Fuentes de Información \\
\hline METO & $\begin{array}{l}\text { Fundamentos y Metodologías de } \\
\text { Investigación. Estudios Métricos de } \\
\text { Información }\end{array}$ \\
\hline PLANI & $\begin{array}{l}\text { Planificación, Organización y } \\
\text { Evaluación de Unidades de } \\
\text { Información }\end{array}$ \\
\hline REPRE & $\begin{array}{l}\text { Representación y Recuperación de } \\
\text { la Información. }\end{array}$ \\
\hline TINFO & $\begin{array}{l}\text { Tecnologías de la Información y } \\
\text { Edición Digital }\end{array}$ \\
\hline
\end{tabular}

Tabla II. Materias obligatorias recogidas en el Libro Blanco de ANECA del Titulo de Grado en Información y Documentación

El método seguido ha sido simple. Se ha analizado la temática de cada trabajo desarrollado y se le ha asignado, por un lado, la materia del plan de estudios antiguo y por el otro, la materia obligatoria del Libro Blanco. Una vez hecho este recuento, se procedió a la obtención de resulta- dos y a la verificación de las hipótesis del estudio.

\section{Resultados}

Tras el recuento y asignación de materias correspondientes al título actual de Licenciado en Documentación a cada uno de los 249 PFC realizados hasta la actualidad, se obtuvieron los siguientes resultados.

\subsection{Distribución de trabajos por materia}

\begin{tabular}{lll}
\hline Materia & Total & $\%$ \\
\hline ESTA & 4 & 1,6 \\
OREMP & 6 & 2,4 \\
PESID & 58 & 23,29 \\
SINFO & 7 & 2,81 \\
SRPAC & 15 & 6,02 \\
TDAI & 9 & 3,61 \\
TIN & 0 & 0,4 \\
TMARI & 14 & 5,62 \\
Tr.(Dipl.) & 59 & 23,69 \\
Op. (Dipl.) & 40 & 16,06 \\
Op. (Lic.) & 36 & 14,45 \\
\hline TOTAL & 249 &
\end{tabular}

Tabla III. Recuento de PFC realizados y materias de Licenciado en Documentación asignadas

Lo cierto es que sorprende enormemente comprobar que tras la materia PESID (Planificación y Evaluación de Servicios de Información Documental) que obtiene el $23,69 \%$ de los PFC realizados, aparece en segundo lugar con un $23,29 \%$ de los proyectos realizados, el conjunto de PFC relacionados con asignaturas troncales de la Diplomatura en Biblioteconomía y Documentación.

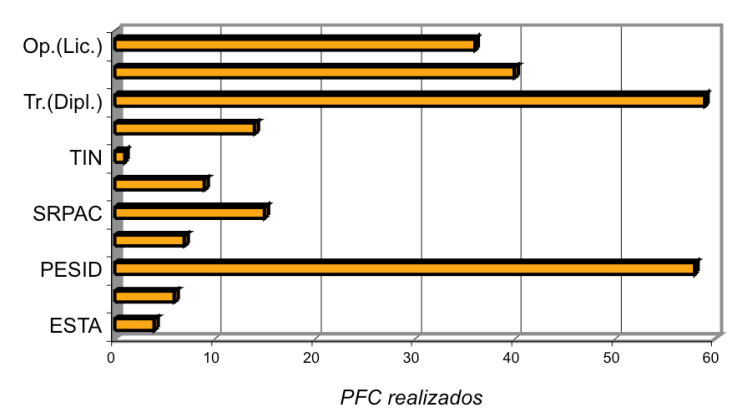

Figura 1. $N^{\circ}$ de PFC realizados en función de las materias de Licenciado en Documentación 
También llama la atención que en tercer y cuarto lugar aparecen las materias optativas del título de Diplomado en Biblioteconomía y Documentación y del título de Licenciado en Documentación. Lo cierto es que sólo una materia troncal del título de Licenciado parece haber despertado suficientemente el interés de los estudiantes para la realización de sus proyectos.

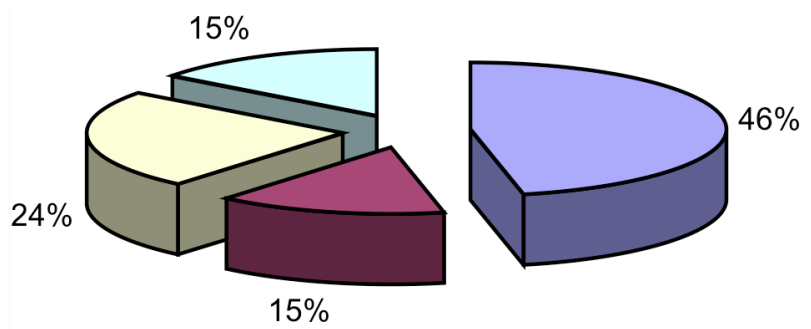

Troncal.Lic $\square$ Optat.Lic $\square$ Troncal.Dip $\square$ Optat.Dip

Figura 2. Distribución porcentual de los PFC realizados por tipo de materia

Lo cierto es que ninguna otra materia troncal del título de Licenciado en Documentación muestra unos valores considerables y quedan muy lejos de la materia de Planificación y Evaluación de Sistemas de Información Documental. También hay que resaltar la escasa presencia de proyectos desarrollados en la materia de Estadística (4 trabajos) o en la materia de Técnicas de Indización.

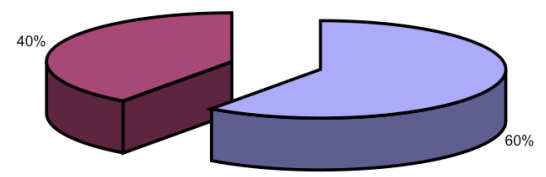

$\square$ Licenciado $\square$ Diplomado

Figura 3. Distribución porcentual de los PFC realizados $x$ titulación

En la tabla IV se muestran los datos obtenidos en relación a las materias obligatorias propuestas en el Libro Blanco del nuevo título de Grado.

Ahora sí se produce una distribución algo más proporcionada entre las materias obligatorias del nuevo título y los temas de interés. Otro factor interesante es que todos los PFC de materias correspondientes a asignaturas optativas del título antiguo han podido asignarse a materias de la nueva propuesta, lo que habla bien de la visión integradora y sintetizadora de la misma. Como puede observarse la materia DOCU (Documentos, Unidades y Sistemas de Informa- ción) es la más tratada con un $32 \%$ de los PFC realizados, seguida de la materia PLANI (Planificación, Organización y Evaluación de Unidades de Información) con un $28 \%$. Por el contrario, la materia METO es la menos tratada en los PFC registrados.

\begin{tabular}{lcc}
\hline Materia & Total & $\%$ \\
\hline ARCHI & 18 & 7,22 \\
DOCU & 80 & 32,12 \\
FUEN & 23 & 9,23 \\
METO & 14 & 5,62 \\
PLANI & 63 & 25,3 \\
REPRE & 29 & 11,64 \\
TINFO & 22 & 8,83 \\
\hline TOTAL & 249 & \\
\hline
\end{tabular}

Tabla IV. Recuento de PFC y materias del Grado en Información y Documentación asignadas

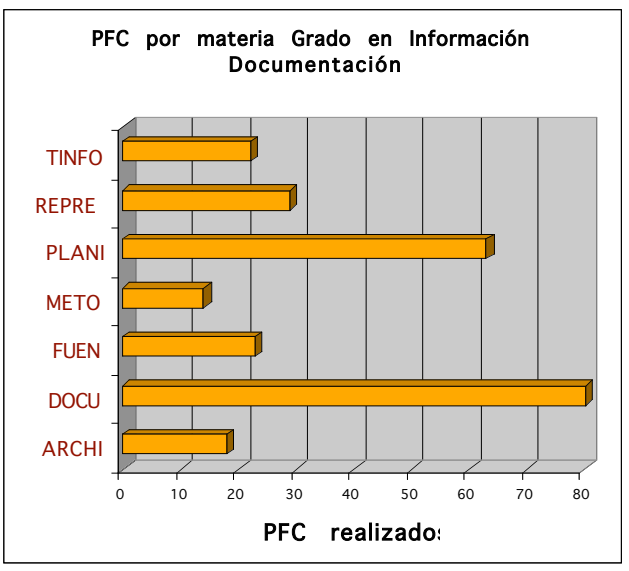

Figura 4. $N^{\circ}$ de $P F C$ realizados en función de las materias de Licenciado en Documentación

La fuerte concentración de trabajos desarrollados en dos materias provoca una serie de desfases entre la importancia relativa que se le ha dado a las mismas dentro del currículo del futuro Graduado y el interés que las mismas han despertado para los estudiantes, tal como se refleja en la siguiente imagen.

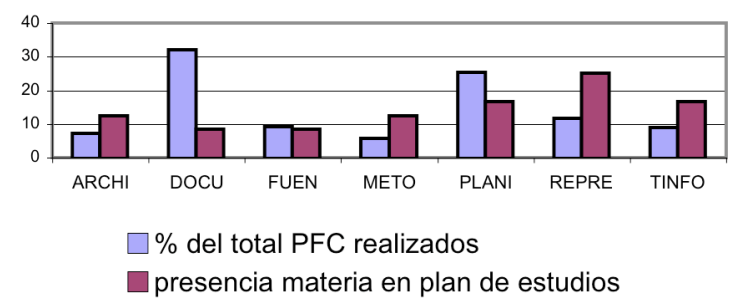

Figura 5. Contraste distribución materias en plan de Grado e interés temático de los PFC realizados 
La concentración indicada anteriormente la que presenta ciertos desajustes entre ambas distribuciones comparadas en la Fig. 4. Sólo en la materia de Fuentes de Información se da un cierto equilibrio entre la propuesta y la realidad y sigue latente una cierta carencia de realización de trabajos en las materias de Análisis y Representación del Conocimiento y en Tecnologías de la Información y Edición Digital.

\subsection{Distribución de trabajos por áreas de conocimiento}

Si analizamos las áreas de conocimiento a la que están adscritos los profesores directores de los PFC realizados, es el área de Biblioteconomía y Documentación la que acapara alrededor del $90 \%$ de la producción. En esta ocasión el resultado es más lógico tanto por la mayor presencia de esta área en la troncalidad del título como en el conjunto de profesores de la Facultad. Aunque la presencia de alguna área sea testimonial, al menos todas ellas han colaborado en la realización de algún proyecto. Hemos de indicar que en esta distribución se ha considerado sólo el área del director del proyecto, no habiéndose computado la del codirector, esta decisión ha perjudicado levemente al área de Ciencias y Técnicas Historiográficas que aumentaría un poco su importancia porcentual.

\begin{tabular}{|c|c|c|c|}
\hline Área & Descripción & Total & $\%$ \\
\hline BIBLIO & $\begin{array}{l}\text { Biblioteconomía y } \\
\text { Documentación }\end{array}$ & 226 & 90,76 \\
\hline ESTA & Estadística & 1 & 0,4 \\
\hline SINFO & $\begin{array}{l}\text { Lenguajes y } \\
\text { Sistemas } \\
\text { Informáticos }\end{array}$ & 7 & 2,81 \\
\hline IA & Inteligencia Artificial & 3 & 1,2 \\
\hline OREMP & $\begin{array}{l}\text { Organización de } \\
\text { Empresas }\end{array}$ & 9 & 3,61 \\
\hline PALEO & $\begin{array}{l}\text { Ciencias y Técnicas } \\
\text { Historiográficas }\end{array}$ & 3 & 1,2 \\
\hline TOTAL & & 249 & \\
\hline
\end{tabular}

Tabla V. Recuento de PFC realizados por área de conocimiento del director

\subsection{Vinculación materia troncal PE / materias Libro Blanco.}

Para finalizar este análisis se han consignado las relaciones entre asignaturas de los dos esquemas de plan de estudio: el oficial vigente de Licenciado en Documentación ( $2^{\circ}$ ciclo) y la propuesta del Título de Graduado en Información y Documentación. El objeto de este análisis es conocer las posibles vinculaciones entre el tratamiento de materias en los TFG potenciales a realizar en los próximos cursos académicos y, por tanto, para poder establecer una planificación diversificada para fomentar las materias menos trabajadas en los futuros TFG.

\begin{tabular}{|c|c|c|c|c|c|c|c|}
\hline & \multicolumn{7}{|c|}{ Graduado en Documentación } \\
\hline & $\begin{array}{l}\text { 叧 } \\
\stackrel{\text { On }}{I} \\
\text { I }\end{array}$ & $\begin{array}{l}\text { 믈 } \\
\text { О } \\
\text { С }\end{array}$ & $\begin{array}{l}\text { ᄁָ } \\
\text { 而 }\end{array}$ & $\frac{3}{3}$ & $\frac{0}{\underline{D}}$ & 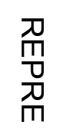 & $\begin{array}{l}\text { 검 } \\
\text { Zn } \\
\text { On }\end{array}$ \\
\hline ESTA & & & & 4 & & & \\
\hline OREMP & 1 & 2 & & & 3 & & \\
\hline PESID & 4 & 12 & 1 & & 35 & 1 & 5 \\
\hline SINFO & & & & & & & 7 \\
\hline SRPAC & & 2 & & & 5 & 5 & 3 \\
\hline TDAI & & & 1 & 8 & & 1 & \\
\hline \multicolumn{8}{|l|}{ TIN } \\
\hline TMARI & & & & & & 12 & 1 \\
\hline Tr.(Dipl.) & 11 & 16 & 16 & 1 & 9 & 5 & \\
\hline Op. (Dipl.) & 2 & 20 & 5 & 1 & 7 & 4 & 4 \\
\hline Op. (Lic.) & & 29 & & & 3 & 1 & 2 \\
\hline
\end{tabular}

El vínculo más fuerte que aparece (en 35 ocasiones, alrededor de un 14\%) es el existente entre las materias PLANI (Graduado). y PESID (Licenciado). Le siguen con un $11 \%$ las materias DOCU (Graduado) y Op. (Lic.), lo que quiere decir que algunas optativas de la Licenciatura encuentran acomodo en una materia obligatoria nueva. Además las asignaturas troncales y optativas del título de Diplomado en Biblioteconomía y Documentación también presentan porcentajes significativos con respecto a DOCU y FUEN (Graduado).

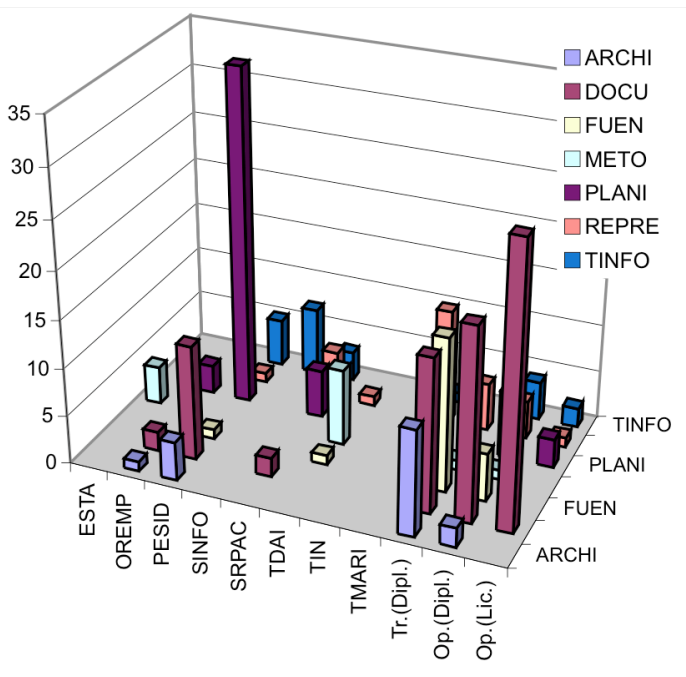

Figura 5. Coincidencias materias planes actual/nuevo 
Existe una desigualdad relevante respecto a unas materias con otras ya que muchas apenas han sido tratadas en proyecto alguno desarrollado hasta ahora. Este hecho debe someterse a reflexión por parte del profesorado para la averiguación de las posibles causas y por tanto de las posibles soluciones.

\section{Conclusiones}

A modo de reflexión general parece adecuado concluir que la experiencia de la Universidad de Murcia conduce a pensar que existe un alto riesgo de elevada concentración de futuros Trabajos Fin de Grado alrededor de algunas materias (Documentos, Unidades y Sistemas de Información y Planificación y Evaluación) dejando bastante al margen a las demás, con excepción quizá de Fuentes de Información. No parece descabellado suponer que cuando este hecho se ha venido produciendo a lo largo de casi una década, pueda trasladarse al nuevo plan de estudios y a otras universidades. Esto tiene que hacer reflexionar a los responsables del desarrollo de dicha asignatura en un futuro inmediato por si introducen algún mecanismo regulador. En teoría debería ser necesario porque la importancia que le da el Real Decreto 1393/2007 a esta asignatura así parece recomendarlo, ya que se corre el riesgo de cargar de trabajo en exceso al profesorado de unas materias.

Centrando el análisis de forma específica en algunos de los resultados ofrecidos por este estudio, se puede aportar la siguiente serie de conclusiones específicas:

1. Es indudable que la materia Planificación, Organización y Evaluación de Unidades de Información es la que más se presta para el desarrollo de un Trabajo de Fin de Grado. Las competencias de la misma se encuentran muy estrechamente vinculadas a la naturaleza de esta actividad, aunque si se profundiza algo más en cuanto al objetivo y alcance de los trabajos se puede observar que la mayoría responden a un patrón genérico aplicado en distintos casos, la mayoría de las veces a nivel teórico, sin intento alguno de plasmarlo posteriormente. Por lo tanto, se trata más de trabajos de "planificación y organización" que de "evaluación". Quizá sería interesante que, en el desarrollo de la futura asignatura, se hiciera especial hincapié en que este tipo de proyectos deberían abarcar la totalidad de las competencias de la asignatura.

2. En el caso particular de nuestra Universidad, muchos estudiantes han realizado proyectos vinculados con conservación y restauración del Patrimonio Documental y también con la Historia del Libro. En el nuevo título de Grado parte de estos contenidos se encuentran en la materia Documentos, Unidades y Sistemas de Información y otros (los de conservación) en la materia Planificación, Organización y Evaluación de Unidades de Información. Si bien puede resultar complicado clasificar un proyecto en cualquiera de estas dos materias, lo que no es tan difícil es afirmar que estas competencias resultan de gran interés para el desarrollo de los futuros TFC.

3. A pesar de que dos materias del nuevo título poseen mayor presencia en la distribución de trabajos realizados, resulta más homogénea y equitativa esta distribución que la de PFC realizados por materia del plan de estudio de Licenciado, donde se presentan desajustes excesivos y nada recomendables, llegando a darse el caso de una materia con un único PFC desarrollado.

4. Ciertamente, es escasa la aportación de algunas de las materias más específicas de nuestra titulación a la realización de los Trabajos de Fin de Grado. Conseguir un mayor equilibrio entre todas las materias creemos debe ser un objetivo a lograr con la puesta en marcha de los nuevos planes de estudio y el reparto de la carga docente en los departamentos universitarios.

5. En nuestro ámbito docente e investigador resulta muy frecuente hallar comentarios sobre la gran interdisciplinariedad de nuestra materia, la Información y la Documentación. Quizá este grado de sinergia con otras disciplinas no se está produciendo con la dirección de los trabajos de fin de carrera, donde nuestra área concentra alrededor del $90 \%$ de los mismos. Sería deseable una mayor colaboración entre todos los profesores vinculados a estos estudios.

\section{Referencias}

ANECA. Libro Blanco del Título de Grado en Información y Documentación. Madrid: ANECA, 2004. http://www.um.es/f-comunicacion/documentos/libro blanco_comunicacion.pdf (Consulta: 10 mayo 2008)

Kajberg, L. and Lorring, L. European Curriculum Reflections on Library and Information Science Education. Copenhagen: The Royal School of Library and Information Science, 2005. http://biblis.db.dk/Archimages/423.12.05. PDF (Consulta: 12 mayo 2008)

Mec. Real decreto 912/1992, de 17 de julio, por el que se establece el titulo universitario oficial de Licenciado en Documentacion y la aprobación de las directrices generales propias de los planes de estudios conducentes a la obtención de aquel. [En línea] http://www.boe.es/g/es/bases_datos/doc.php?coleccion=iberlex\&id $=1992 / 20$ 359 (Consulta: 18 junio 2008). 
Mec. Real decreto 1393/2007, de 30 de octubre, por el que se establece la ordenación de las enseñanzas universitarias oficiales. http://www.boe.es/g/es/bases_datos/ doc.php?coleccion=iberlex\&id=2007/18770\&txtlen=1000 (Consulta: 10 junio 2008).

Universidad de Murcia, Facultad de Comunicación y Documentación. Reglamento del PFC de Licenciado en Documentación. Murcia: Universidad, 2000. http://www.um.es/f-comunicacion/pfcs/pfc-docu/index.php (Consulta: 06 junio 2008).

Universidad de Murcia. Resolución de 18 de octubre de 2000, de la Universidad de Murcia, por la que se hace pública la modificación del plan de estudios de Licenciado en Documentación. [En línea] Madrid: Boletín Oficial del Estado, 2000. http://www.boe.es/boe/dias/2000/11/10/pdfs/A39367-39368.pdf (Consulta: 12 mayo 2008) 